\title{
Dynamic Response of Two-Electrode Distributed Feedback Laser for Stable Signal Mode Operation
}

\author{
H. Bousseta*, A. Zatni*, A. Amghar**, A. Moumen*, A. Elyamani* \\ * M.S.I.T Laboratory, Department of Computer Engineering, high school of technology, Ibn Zohr University, Agadir \\ Morocco \\ ** Department of physics, faculty of sciences, Ibn Zohr University, Agadir Morocco
}

\begin{tabular}{|c|c|}
\hline Article Info & ABSTRACT \\
\hline Article history: & \multirow{9}{*}{$\begin{array}{l}\text { The longitudinal spatial hole burning (LSHB) effect has been known to limit } \\
\text { the performance of distributed feedback (DFB) semiconductor lasers to } \\
\text { achieve a better dynamic signal mode operation (DSMO). So, in order to } \\
\text { ensure a stable (DSMO), we propose a novel device design of two electrode } \\
\text { DFB lasers with longitudinal variation in the coupling coefficient (distributed } \\
\text { coupling coefficient (DCC)), the structure also contains a phase shifted in } \\
\text { middle of the cavity. By means of the finite difference time domain (FDTD) } \\
\text { numerical method, we analyze dynamic response of our structure and we also } \\
\text { compare the results with the conventional two electrode DFB laser (TE- } \\
\text { DFB). The numerical simulation shows that, a better dynamic signal mode } \\
\text { has been achieved by TE-DCC-DFB lasers in comparison with TE-DFB laser } \\
\text { due to its better and high side mode suppression ratio (SMSR). Therefore, the } \\
\text { TE-DCC-DFB lasers will be useful to extend the transmission distance in } \\
\text { optical fiber communication systems. }\end{array}$} \\
\hline Received Sep 8, 2014 & \\
\hline Revised Nov 13, 2014 & \\
\hline Accepted Dec 10, 2014 & \\
\hline Keyword: & \\
\hline Distributed feedback lasers & \\
\hline Dynamic response & \\
\hline Finite difference time domain & \\
\hline Numerical simulation & \\
\hline
\end{tabular}

Copyright (C) 2015 Institute of Advanced Engineering and Science. All rights reserved.

\section{Corresponding Author:}

Hamza Bousseta,

M.S.I.T Laboratory, Department of Computer Engineering, high school of technology, Ibn Zohr University, Agadir Morocco.

Email: Hamza.bousseta@gmail.com

\section{INTRODUCTION}

Advanced semiconductor laser are key devices in high speed modern optical communication systems [1-7]. Among many different laser structures, the distributed feedback (DFB) structure has been widely used in semiconductor lasers to achieve a stable DSMO due to their small size, high optical output power, fast response and low threshold current [8-12]. Therefore, Introducinga $\lambda / 4$ phase shift in the grating structure is effective for achieving stable signal mode operation because of high side mode suppression ratio [13-14]. However, the presence of the phase shift when the coupling coefficient is large or at higher injection currents, generally causes spatial non uniformities of photon and carrier densities or effective index along the cavity[15], this phenomenon called spatial hole burning (SHB) effect[16], This SHB is found to enhance the side mode.

Usually a variety of methods can be used to solve this issue, the first one consisted in enlarging the threshold gain margin by introducing gain coupling mechanism or constructing distributed coupling coefficient gratin (DCC-DFB) [17-20], the second is to weaken the no uniform distribution of carriers, for example they can be obtained by utilizing multiple discrete phase shift (MPS-DFB) [21] or by introducing longitudinal chirped grating for bragg period [5], [22] and [23].

In our previous work, we propose a two electrode DFB laser with stronger centre coupling and a $\lambda / 4$ phase shift in the center of the cavity (TE-DCC-DFB), this structure is another way to improve the output parameters such as SMSR and power output. So, in this paper, we have simulated the dynamic 
parameters of TE-DCC-DFB laser and compared it with conventional TE-DFB laser in order to show the superiority of our structure.

The remainder of this paper is organized as follow: time domain model including the coupled model equations and numerical simulations method are briefly described in section 2. Simulations results of proposed structure, namely those concerning dynamic characteristics in section 3. Finally, we closed the paper by a brief conclusion in section 4 .

\section{THEORY AND DESCRIPTION OF MODEL}

The laser structure analyzed in our model is depicted schematically on Figure 1. The structure is related to two electrodes, the first electrode extend from $z=0$ to $z=L / 2$. Contrariwise the second electrode, from $z=L / 2$ to $z=L$, the bias current $I_{A}$ and $I_{B}$ are injected independently into the cavity. The structure is divided into four sections, the length of the center and side sections is $\mathrm{L}_{c}$ and $\mathrm{L}_{\mathrm{s}}\left(\mathrm{L}_{\mathrm{c}(\mathrm{s})}\right.$ is the length of the section with coupling coefficient $\left.k_{c(s)}\right)$, respectively. The normalized coupling coefficient of side sections and of center sections are $k_{s} L_{s}$ and $k_{c} L_{c}$, respectively.

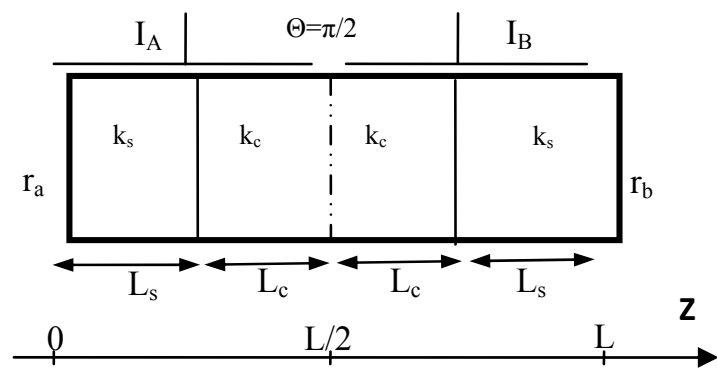

Figure 1. Schematic diagram of TE-DCC-DFB laser

Taking into account the longitudinal change of the coupling coefficient in the structure i.e $k$ is $\mathrm{z}$ dependent. Thus, for TE-DCC-DFB laser, the coupling coefficient ratio as:

$$
r=\frac{k_{c} L_{c}}{k_{s} L_{s}}=\frac{0.75}{0.33}
$$

with

$$
k L_{\text {Total }} \text { defined as } k L_{\text {Total }}=2\left(k_{s} L_{s}+k_{c} L_{c}\right)
$$

The spatiotemporal dynamic of the DFB is characterized by the carrier number density $N$ and the electric field of

$$
E^{\text {total }}(z, t)=\left[F(z, t) e^{-i \beta_{0} z}+B(z, t) e^{i \beta_{0} z}\right] e^{i \omega_{0} t}
$$

Where $F$ and $B$ represent the slowly varying envelopes of the forward and backward waves, which are coupled through the laser structure and $\omega_{0}$ is the reference frequency. $\beta_{0}$ is the propagation constant at bragg frequency, given by $\beta_{0}=\pi / \Lambda$, with $\Lambda$ is the period of the grating

The rate equations of the carrier density and time dependent coupled wave equations of the slowly varying envelop are given by [17]:

$$
\frac{d N(z, t)}{d t}=\frac{I_{a, b}}{q V}-\frac{N(z, t)}{\tau}-B N^{2}(z, t)-C N^{3}(z, t)-\frac{C_{g} A_{o}\left(N(z, t)-N_{o}\right) P(z, t)}{1+\varepsilon P(z, t)}
$$




$$
\begin{aligned}
& \left(\frac{1}{C_{g}} \frac{\delta}{\delta t}+\frac{\delta}{\delta z}\right) F(z, t)=\left(g(z, t)-\alpha_{s}-i \delta(z, t)\right) e^{-i \theta(z)} F(z, t)+i k(z) e^{-i \theta(z)} B(z, t)+G(z, t) \\
& \left(\frac{1}{C_{g}} \frac{\delta}{\delta t}-\frac{\delta}{\delta z}\right) B(z, t)=\left(g(z, t)-\alpha_{s}-i \delta(z, t)\right) e^{i \theta(z)} B(z, t)+i k(z) e^{i \theta(z)} F(z, t)+G(z, t)
\end{aligned}
$$

With $C_{g}$ is the group velocity, $\alpha_{s}$ is the optical loss coefficient, $q$ is the electron charge, the parameter $\tau$ stands for the electrons lifetime, $V$ is the cavity volume, $\varepsilon$ is a non-linear coefficient to take into account saturation effects, $k(z)$ is the coupling coefficient. Also, $I_{A, B}$ is the uniform current bias of electrode denoted by the subscript, $B$ is the bimolecular recombination coefficient, $C$ is the Auger recombination coefficient, $\theta(z)$ is the phase shift at $z$ position, $N(z, t)$ is the carrier density, $N_{0}$ is the carrier density at transparency and $P(z, t)=|F(z, t)|^{2}+|B(z, t)|^{2} \quad(7)$ is the photon density [17]. $g(z, t)$ is the material gain, given by [17]:

$$
g(z, t)=\frac{\Gamma A_{o}\left(N(z, t)-N_{o}\right)}{2(1+\varepsilon P(z, t))}
$$

Where $\Gamma$ is the optical confinement factor, $A_{0}$ is the differential gain. The $\delta(z, t)$ represent the frequency detuning defined as [17]:

$$
\delta(z, t)=\frac{2 \pi}{\lambda_{o}} n_{e f f}(z, t)-\frac{\pi}{\Lambda}
$$

With $\lambda_{0}$ is an approximate emission wavelength. The effective refractive index can be expressed as [17]:

$$
n_{e f f}(z, t)=n_{e f f}^{o}-\frac{\Gamma \alpha_{H} A_{o} \lambda_{o}}{4 \pi}\left(N(z, t)-N_{o}\right)
$$

Where $n_{e f f}^{o}$ is the effective index at transparency and $\alpha_{H}$ represent the phase amplitude coupling factor.

The spontaneous emission fields coupled into the forward and backward waves are $G$, thus the autocorrelation function defines as [17]:

$$
\left\langle G(z, t) G^{*}\left(z^{\prime}, t^{\prime}\right)\right\rangle=\frac{\beta K B N^{2}(z, t) \delta\left(t-t^{\prime}\right) \delta\left(z-z^{\prime}\right)}{C_{g} L}
$$

Where $\beta$ is the spontaneous coupling factor, $K$ is the Petermann coefficient.

For a computerized calculation, the coupled equations (5) and (6) are solved numerically using the finite difference time domain (FDTD)[24], then this method is based on solving the coupled wave equations in the time domain by a first order difference approximation to the partial difference[24-25]. So, we can showed that:

$$
\begin{aligned}
& F(t+\delta t, z+\delta z)-F(t, z)=\Delta z\left[\left(\left(g(z, t)-\alpha_{s}-i \delta(z, t)\right) \times F(t, z)+i k(z) B(t, z) e^{i \theta(z)}+G(t, z)\right]\right. \\
& B(t+\delta t, z+\delta z)-B(t, z)=\Delta z\left[\left(\left(g(z, t)-\alpha_{s}-i \delta(z, t)\right) \times B(t, z)+i k(z) F(t, z) e^{-i \theta(z)}+G(t, z)\right]\right.
\end{aligned}
$$

The FDTD method can be utilized to simulate the dynamic responses of the DFB laser by solving the coupled equations (12) and (13). Therefore, in this work we have developed a FDTD algorithm, which has been applied to those equations. The numerical procedure of this method involves dividing the cavity length into several uniform grating sub-sections $S=200$ of equal length $\Delta z=L / S=C_{g} \cdot \Delta t$.

Dynamic Response of Two-Electrode Distributed Feedback Laser for Stable Signal Mode ... (H. Bousseta) 


\section{NUMERICAL RESULTS AND DISCUSSIONS}

The main goal of this paper is the simultaneous assessment of dynamic characteristics of TE-DCCDFB laser structure and the comparison with the conventional TE-DFB laser structure, Table 1 shows the parameters used in these simulations. In the following discussion the performance comparison between the two structures under various system parameters will be previewed in detail.

First, we present the dynamic representation of the laser output power, Figure 2.a and b shows the evolution of the photon density as a function of time at $z=L$ and also illustrates the optical output spectrum for the conventional TE-DFB laser. Both sections are biased sufficiently as $I_{A}=86 \mathrm{~mA}$ for section A (left section $L_{s}+$ left section $L_{C}$ ) and $I_{B}=75 \mathrm{~mA}$ for section B (right section $L_{C}+$ right section $L_{s}$ )

To facilitate comparison, the transient response and the optical output spectrum of the TE-DCCDFB laser are also show in Figure 3.a and b. the optical spectrum is obtained by performing fast Fourier transform (FFT) for optical output field within $[3,4]$ ns.

Table 1. parameter values used in simulations

\begin{tabular}{ccc}
\hline parameters & symbol & value \\
\hline Linear recombinaison & $\tau$ & $4.10^{-9} \mathrm{~s}^{-1}$ \\
Bimolicular recombinaison & $B$ & $10^{16} \mathrm{~m}^{-3} \mathrm{~s}^{-1}$ \\
Auger recombinaison & $C$ & $3.10^{-41} \mathrm{~m}^{-6} \mathrm{~s}^{-1}$ \\
Differential gain & $A_{0}$ & $2,7.10^{-20} \mathrm{~m}^{2}$ \\
Internal loss & $\alpha_{S}$ & $3000 \mathrm{~m}^{-1}$ \\
Effective index transparency & $n^{0}$ & 3.2 \\
transparency Carrier density & $N_{0}$ & $1.10^{24} \mathrm{~m}^{-3}$ \\
Linewidth enhancement factorc & $\alpha_{H}$ & 5,4 \\
Group velocity & $C_{g}$ & $3.10^{8} / 3,7 \mathrm{~ms}^{-1}$ \\
Petermann factor & $K$ & 1 \\
Peak wavelength at transparency & $\lambda_{0}$ & $1,5648.10^{-6} \mathrm{~m}$ \\
Optical confinement factor & $\Gamma$ & 0.35 \\
Grating period & $\Lambda$ & $227.039 .10^{-9} \mathrm{~m}$ \\
Non linear gain coefficient & $\varepsilon$ & $1,5.10^{-23}$ \\
Lsaser cavity length & $L$ & $500.10^{-6} \mathrm{~m}$ \\
Volume for active region & $V$ & $90.10^{-6} \mathrm{~m}$ \\
Spontaneous coupling factor & $\beta$ & $5.10^{-5}$ \\
group index & $n_{g}$ & 3.7 \\
& &
\end{tabular}

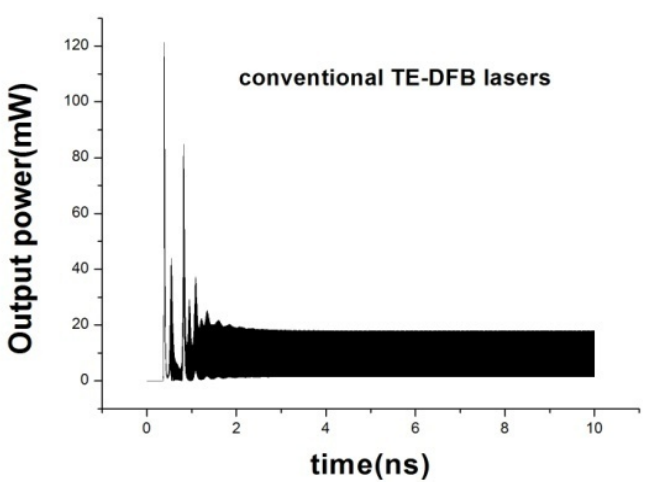

(a)

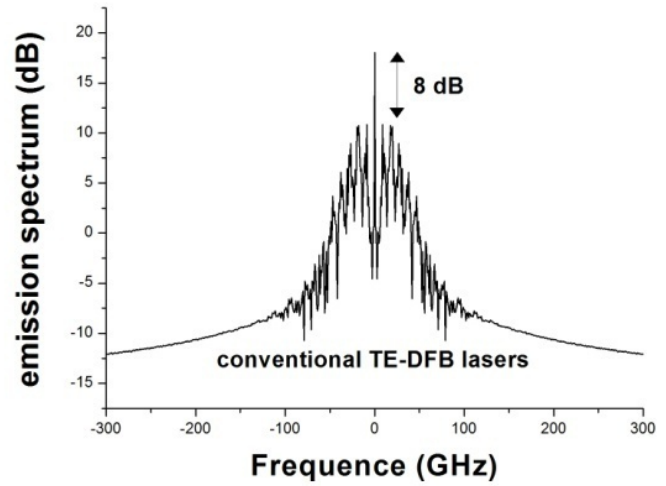

(b)

Figure 2. (a) Transient response $\left(I_{A}=86 \mathrm{~mA}\right.$ and $\left.I_{B}=75 \mathrm{~mA}\right)$ and (b) Output optical spectrum for conventional TE-DFB lasers 
However, a careful study of Figures 2.a and 3.a show that, the lasing output of the conventional TEDFB laser starts after passing needed time to satisfy the threshold condition and it starts with strong amplitude as the consequence of beating between two modes.For TE-DCC-DFB laser, the lasing output starts to oscillate in small amplitude and after approximately $\mathrm{t}=1 \mathrm{~s}$, stable oscillations are observed. Then, it can be observed in the Figure 2.b and 3.b, for the conventional TE-DFB laser, the existence of different frequencies. This is because the effect of the modes beating in optical power [17]. Also there are side modes in addition to the main one and their amplitudes are comparable to the main mode and they cannot be ignored, also the SMSR is estimated to be $8 \mathrm{~dB}$. Furthermore, for the other structure the SMSR is more than $43 \mathrm{~dB}$ due to the single mode output power, the mode beating came from the onset of side mode in the cavity as the result of LSHB [17]. Indeed The conventional TE-DFB laser cavity is rapidly subjected to LSHB, but the TE-DCCDFB laser cavity seems to be widely prevented against it.

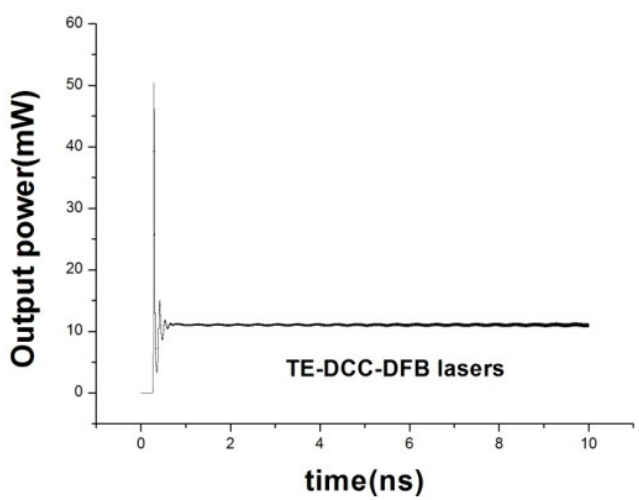

(a)

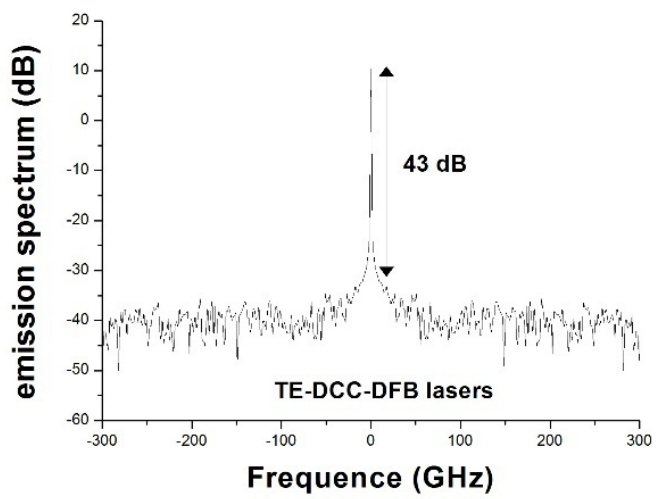

(b)

Figure 3. Longitudinal (a) Transient response ( $I_{A}=86 \mathrm{~mA}$ and $I_{B}=75 \mathrm{~mA}$ ) and (b) Output optical spectrum for TE-DCC-DFB lasers.

In order to understand the effects of LSHB on the power along the cavity, the Figure 4.a shows the magnitude of forward, backward and total internal power along the cavity for TE-DFB laser and for comparisons, we also show the case of conventional TE-DCC-DFB laser structure in Figure 4.b

It is obvious from both figures that the internal optical power increased at the middle of the structure. Hence, for the TE-DCC-DFB the optical output power is increased in right of cavity to $11 \mathrm{~mW}$ i.e higher power output compared to theother structure, the reason is that more photons accumulated at the right facet of cavity [25].

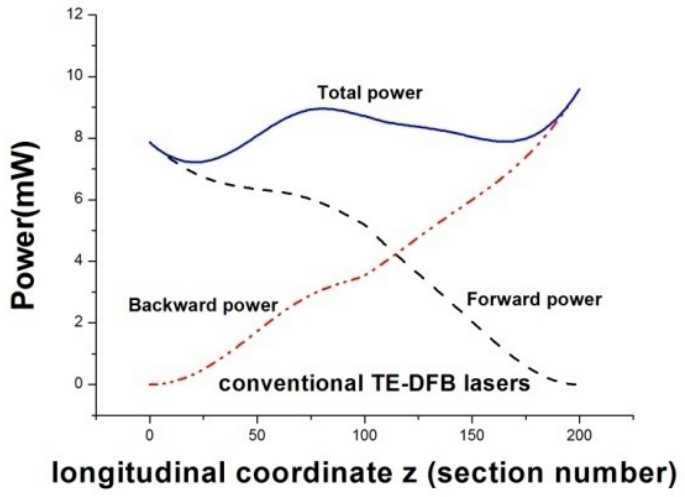

(a)

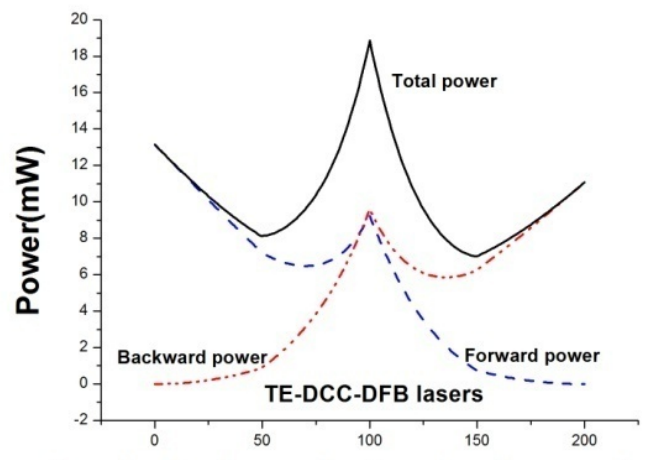

longitudinal coordinate $\mathbf{z}$ (section number)

(b)

Figure 4. Wavelength Forward, backward and total internal power along the cavity. (a) TE-DFB lasers and (b) conventional TE-DCC-DFB lasers. 
The Longitudinal profiles of carrier density and power can also indicate the occurrence of multi mode operation in lasers structures. Hence, the Figure 5 displays the Longitudinal profiles of carrier density in different moments for the twostructures, the first one is taken at $t=1$ ns i.e before the modes beating and the second at $\mathrm{t}=2$ ns i.e during the beating.

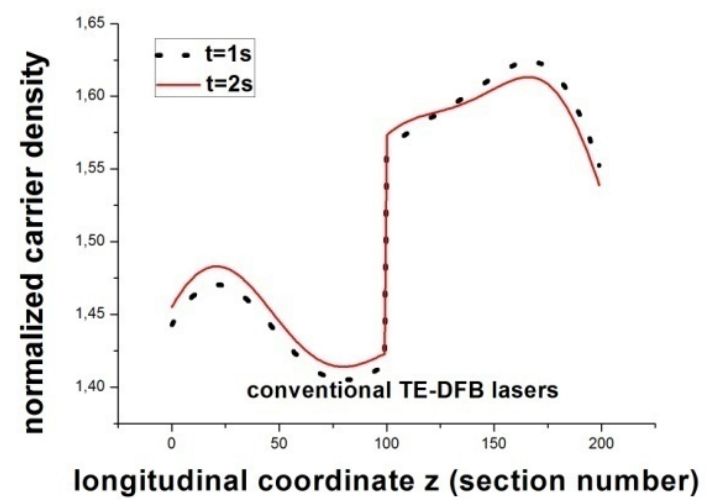

(a)

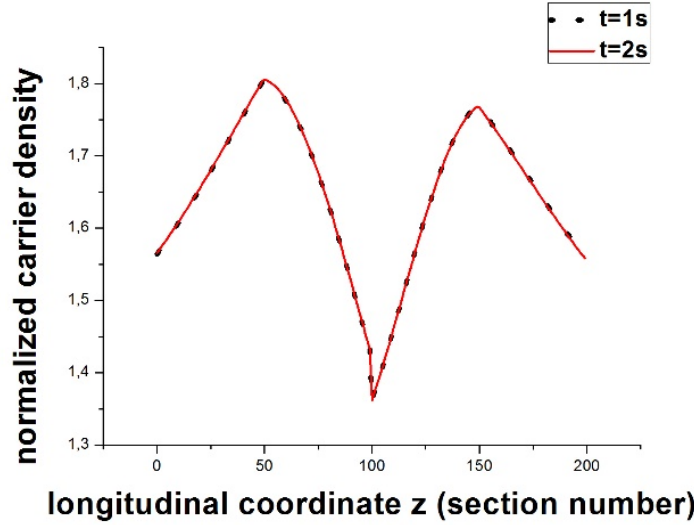

(b)

Figure 5. Longitudinal carrier density profiles in two different instants of the transient response of (a) conventional DFB lasers and (b) TE-DCC-DFB lasers.

We see that, for both structures, there is a discontinuity of the carrier density at the middle of the cavity (the interface between the left and right electrode). This discontinuity is reasonable as long as there is sufficient resistance between these two sections A and B [25]. For the conventional TE-DFB laser, it is observed that the carrier profile is modified between the two instants, this is because of the occurrence of a second mode in the cavity. In contrast for the TE-DCC-DFB laser structure, the carrier density longitudinal profile remains clamped.

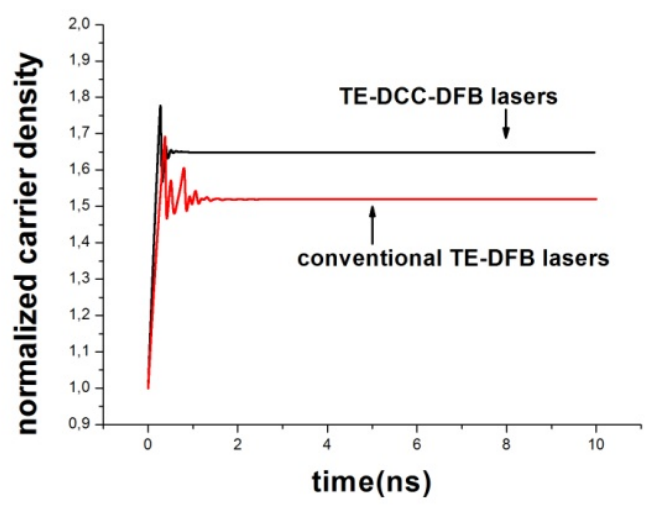

Figure 6. Normalized carrier density versus time for conventional TE-DFB and TE-DCC-DFB. With injection currents $I_{A}=86 m A$ and $I_{B}=75 m A$

Now, In the Figure 6 we present this Normalized carrier density as a function of time.It is obvious from this figure, that the dumping of transient of the TE-DCC-DFB laser is better than for the conventional TE-DFB laser structure and also, the first structure stabilized at $\mathrm{t}=0,8 \mathrm{~ns}$, however the second stabilized at $\mathrm{t}=1.75 \mathrm{~ns}$.Also we observed that the value of $N$ in TE-DCC-DFB laser after stabilization is much less than the other structure, the reason for this phenomenon can be explained in [26]. Finally, to show the effects of the change structure's parameters on side mode suppression ratio (SMSR), Figure 7 displays a comparison between the two structures. We see clearly that, when the biasing current is less than $I_{B}=30 \mathrm{~mA}$, the both 
structure manifest a good SMSR. However, when the biasing is above $30 \mathrm{~mA}$, the SMSR for the conventional TE-DFB laser degraded rapidly to a minimum value equal to $10 \mathrm{~dB}$. In contrast to TE-DCC-DFB laser, the good signal mode operation can be maintained significantly within brad current rage, the maximum values of SMSR reach is $45 \mathrm{~dB}$. The results of this figure shows that, the TE-DCC-DFB laser has given a better transient SMSR, this indicates that the LSHB also plays a role in SMSR [27-28].

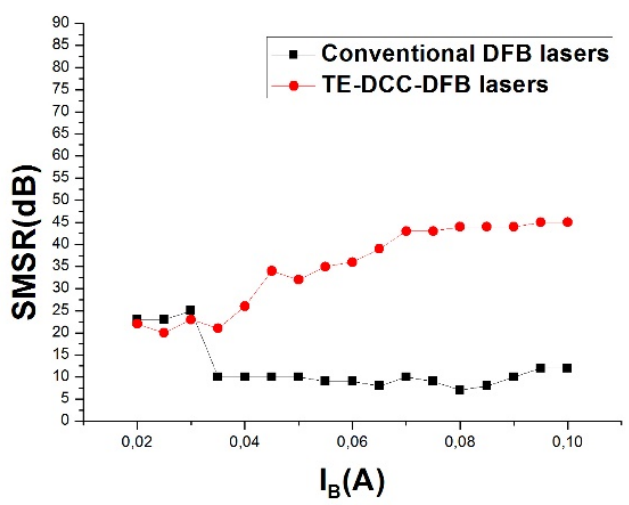

Figure 7. Dependence of SMSR on biasing current for the conventional TE-DFB lasers and TE-DCC-DFB lasers.

\section{CONCLUSION}

In this paper and with the help of a computer algorithm based on the FDTD model, we have presented a traveling wave large signal simulations of dynamic characteristics of TE-DCC-DFB laser. The spontaneous emission, spatial hole burning, longitudinal variation of carrier and photon densities have been taken into consideration in the modeling. The conventional TE-DFB laser which is characterized by its uniform coupling coefficient along the cavity, and the TE-DCC-DFB laser with a $\lambda / 4$ phase shift in center cavity and stronger center coupling coefficient have been investigated and compared.On the other side, the results of simulations showed that, the first structure is not in single mode operation, but the second one acts as a single frequency source with an output power equal to $11 \mathrm{~mW}$ and SMSR will be more than $45 \mathrm{~dB}$. In addition the characteristics of TE-DCC-DFB laser structure have improved the signal mode stability. Therefore the results shows that the best dynamics signal mode can be achieved by the TE-DCC-DFB lasers.

\section{REFERENCES}

[1] Abedelkarim Zatni, et.al. Study of the short pulse generation of the three quarter wave shift DFB laser (3QWSDFB). Annals of Telecommunications. 2005; 60: 698-718.

[2] A. Zatni, J. Le bihan. Analysis of FM and AM responses of a tunable three-electrode DBR laser diode. IEEE journal of Quantum Electronics. 1995; 31: 1009-1014.

[3] D. Ghodsi Nahri, H. Arabshahi. Static Characterization of InAs/AlGaAs Broadband Self-Assembled Quantum Dot Lasers. TELKOMNIKA Indonesian Journal of Electrical Engineering. 2012; 10(1): 55-60.

[4] Ghulam Mehdi, et.al. On the Design of Laser Structured Ka Band Multi-Chip Module. TELKOMNIKA Indonesian Journal of Electrical Engineering. 2014; 12(2): 1250-1257.

[5] Xin-Hong Jia a, et.al. Dynamic single-mode and modulation characteristics analyses for $\lambda / 4$ phase-shifted distributed feedback lasers with chirped grating. Optics Communications. 2007; 279: 356-363.

[6] H.Bousseta, et.al. Static analysis of wavelength tuning in two section index coupled DFB lasers using the transfer matrix method. Journal of Theoretical and Applied Information Technology. 2013; 47(1):10-17.

[7] A.Moumen, et.al. a novel design of quarter wave-shifted distributed feedback laser for high-power single-mode operation. Journal of Theoretical and Applied Information Technology. 2012; 38(2): 210-218.

[8] T. Fessant. Threshold and above-threshold analysis of corrugation-pitch-modulated DFB lasers with inhomogeneous coupling coefficient. IEE ProcOptoelectron. 1997; 144: 365-376.

[9] José A.P. Morgado, Carlos A.F Fernandesand Jose B. M. Bovida. Novel DFB laser structure suitable for stable single longitudinal mode operation. Optics Laser Technology. 2010; 42: 975-984.

[10] Zatni, D. Khatib, M. Bour J. Le bihan. Analysis of the spectral stability of the three phase shift DFB laser (3PSDFB). ANNALS OF TELECOMMUNICATIONS. 2004; 59: 1031-1044.

[11] Carlos Alberto Ferreira Fernandes. stability in single longitudinal mode operation in DFB laser structures. IEEE melecon, May 12-15, Dubrovnik, Croatia, 2004; 3-6. 
[12] N. Bazhdanzadeh, et.al. Dynamic response of QWS-DFB lasers with convex tapered grating structure and non-zero facet reflection. Solid-State Electronics. 2008; 52: 221-226.

[13] Yuechun Shi, et.al. Improved $\lambda / 4$ phase-shifted DFB semiconductor laser with spatial hole burning compensation using grating chirp. Optics Laser Technology. 2012; 44: 2443-2448.

[14] James E. A. Whiteaway, et.al. The design and assessment of $\lambda / 4$ phase-shifted DFB Laser Structures. IEEE journal of quantum electronics.1989; 25(6): 1261-1279.

[15] AbedelkarimMoumen, et.al. Time-domain large signal investigation on dynamic responses of the GDCC quarterly wavelength shifted distributed feedback semiconductor laser. International journal of advanced computer sciences and applications, 2012; 3(9): 165-170.

[16] M. Aliannezhadi, F. Shahshahaniand V. Ahmadi. Improved performance of complex gain-coupled DFB laser by using tapered grating structure. Opt Quant Electron. 2012; 44: 1-16.

[17] Thierry fessant. Influence of a nonuniform coupling coefficient on the static and large signal dynamic behavior of bragg-detuned DFB lasers. Journal of lightwave technology. 1998; 16: 419-427.

[18] T. Fessant, "Enhanced dynamics of QWS-DFB lasers by longitudinal variation of their coupling coefficient. IEEE Photon. Technol. Lett. 1997; 9: 1075-1077.

[19] Xin-Hong Jia, et.al. Detailed modulation response analyses on enhanced signal-mode QWS-DFB lasers with distributed coupling coefficient. Optics communications. 2007; 277: 166-173.

[20] B.S.K. Lo, and H. Ghafouri-shiraz. Spectral characteristics of distributed feedback laser diodes with distributed coupling coefficient, Journal of lightwave technology. 1995; 13(2).

[21] Henning Olesen, et.al. Singal-mode stability of DFB lasers with longitudinal Bragg Detuning. IEEE Photon. Technol. Lett, 1995; 7(5): 461-463.

[22] Yitang Dai and Jianping Yao. Numerical Study of a DFB Semiconductor Laser and Laser Array with Chirped Structure Based on the Equivalent Chirp Technology. IEEE journal of quantum electronics. 2008; 44(10): 938-945.

[23] HartmutHillmer, et.al. Tailored DFB Laser Properties by Individually Chirped Gratings Using Bent Waveguides. IEEE journal of selected topics in quantum electronics. 1995; 1(2): 356-362.

[24] L. M. Zhang, et.al. Dynamic analysis of radiation and side-mode suppression in an second-order DFB laser using time-domain large-signal traveling wave model. IEEE J. Quantum. Electron. 1994; 30:1389-1395.

[25] Xinhong Wang, et.al. Spatiotemporal dynamics and high-frequency self-pulsations in two-section distributed feedback lasers. J. Opt. Soc. Am. B, 1999; 16(11): 2030-2039.

[26] L.M. Zhang, John E. Carroll, and C. Tsang. Dynamic response of the gain coupled DFB laser. IEEE journal of Quantum Electronics, 1993; 29:1722-1727.

[27] T.Fessant. threshold and above-threshold analysis of corrugation-pitch-modulated DFB lasers with inhomogeneous coupling coefficient. IEE Proceeding Optoelectrom. 1997; 144(6): 365-376.

[28] Jianyao Chen, et.al. Self-consistent analysis of side-mode suppression in gain-coupled DFB Semiconductor Lasers. IEEE journal of quantum electronics. 1998; 34(1): 101-109.

\section{BIOGRAPHIES OF AUTHORS}

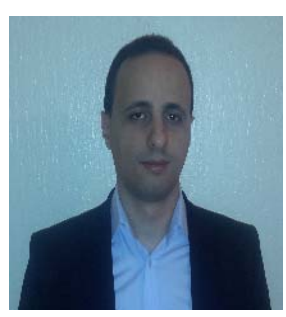

Hamza. BOUSSETA received the MSc degree in electronics, and communication system engineering from faculty of sciences University Mohamed 1 in 2009; he is currently working the $\mathrm{PhD}$ at the centre of doctoral studies (Ibnou Zohr CED). His research interests include design, characterization, modelling and optimization of optoelectronic components and communications systems.

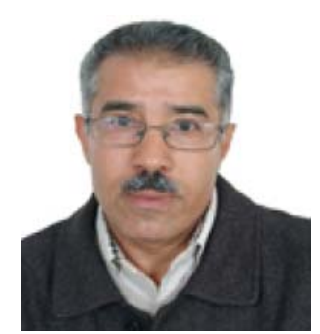

Abdelkarim. ZATNI was educated at the Telecom Bretagne University France; He obtained a $\mathrm{PhD}$ at the National School of Engineers of Brest France in 1994. He has been teaching experience for 22 years. He is currently a Professor and the Head of computer science department in Ibnou Zohr University at Higher School of technology Agadir, Morocco; He conducts his research and teaches in computer science. 